\section{Patient-Centred Care through Storytelling}

In February 2017, the Canadian Society of Hospital Pharmacists (CSHP) launched the Excellence in Hospital Pharmacy program, a strategic initiative designed to improve patient health outcomes. ${ }^{1}$ Of the 3 priority themes of this program, I believe the most vital is patient engagement/patient-centred care. As technology advances, it seems that we are becoming less patient-centred. We now make most treatment decisions with a computer, not the patient. We depersonalize patients by reducing them to a list of diagnoses or a room number. When patients are admitted to hospital, we essentially infringe upon their basic human rights by removing their independence, privacy, dignity, and control, not to mention their clothes. To be patient-centred, we have to acknowledge what it is like to be a patient. I believe the most compelling way to gain this perspective is through storytelling.

Storytelling involves the lesser-known EBM: emotion-based medicine. A patient's description of an encounter with the health care system can be more impactful than a landmark clinical trial, because it forces us to share the patient's emotional experience. What may be routine for us, as health care providers, is often frightening and incomprehensible to patients and/or their families. Storytelling is about the human experience, and telling one's own story is not an easy thing to do-it can conjure feelings of vulnerability, insecurity, and self-doubt. However, we must embrace a culture that empowers patients to share their stories. The 2016 CSHP position statements on pharmacy practice in hospitals and other collaborative health care settings state that the provision of patient-centred care should involve "listening to, understanding, and respecting the patient's story about experiences and expectations that will affect the use of medications."

Health care is complex, and patients routinely feel anxious and insignificant when faced with the impatience, apathy, and condescension that occasionally contaminate our overburdened system. Thus, we sometimes need to be reminded that caring is a fundamental aspect of being a health care professional, and that patients commonly perceive the quality of their care not in terms of technical minutiae, which they may or may not be qualified to judge, but in terms of the ability of the health care team to meet their needs in a respectful manner. To quote Jeff Whissell, a pharmacist colleague from Edmonton, "Patients won't remember the dosage change you make, but they are going to remember how you made them feel."

I was struck by the power of storytelling as a means to promote patient-centredness at a CSHP Alberta Branch continuing education event in 2014. Sue Robins, a writer and speaker specializing in patient and family engagement, shared her personal experiences with the health care system. Sue's aims are to motivate, inspire, and educate for positive change in health care, and she advocates that patients do better when they themselves feel cared for.

We are taught empathy in our pharmacy training, but I do not think one can really appreciate the patient experience without having personally accessed the health care system. When my son was born, my wife had a potentially life-threatening complication, which was by far the most terrifying incident of our lives. In the 3 years since, my son, like most children, has been to the emergency department at our local hospital multiple timesfor fifth disease, croup, and otitis media (to name a few of his diagnoses) — and each time my persona as the objective, rational health care professional has been replaced by that of the anxious, irrational father. I am lucky, in that both my wife and our son are healthy, but through these interactions I have realized first-hand that most negative interactions between patients/families and health care providers are derived from fear and stress. When I access the health care system, I am unapologetically abrasive toward the "business-as-usual" approach, but soften to deference and appreciation with the smallest demonstration of care. These experiences have precipitated new-found respect for my patients. I now make more of an effort to demonstrate empathy in simple and practical ways with the goal of, if nothing else, reassuring the patient that we (i.e., the health care team) are there to take care of them and support their active participation in decision-making.

Despite our technologies, advanced skills, and scientific evidence, health care, at its core, is about providing care, which is 
rooted in emotion. And there is no better way to evoke emotion than a good story. When you provide care to your patients, I encourage you to avoid focusing just on their problems-instead, focus on each patient's story.

\section{References}

1. Excellence in Hospital Pharmacy program. Ottawa (ON): Canadian Society of Hospital Pharmacists; 2016 [cited 2017 Jul 11]. Available from: https:// www.cshp.ca/excellence

2. Pharmacy practice in hospitals and other collaborative healthcare settings: position statements. Ottawa (ON): Canadian Society of Hospital Pharmacists; 2016 [cited 2017 Jul 11]. Available from: https://cshp.ca/sites/default/files/ files/publications/PharmacyPractice.pdf

Arden R Barry, BSC, BSc(Pharm), PharmD, ACPR

Clinical Pharmacy and Research Specialist

Lower Mainland Pharmacy Services

Primary Care Clinic, Chilliwack General Hospital

Chilliwack, British Columbia

Arden Barry is also an Assistant Professor (Partner) with the Faculty of Pharmaceutical Sciences, The University of British Columbia, Vancouver, British Columbia.

Competing interests: None declared.

Acknowledgement: The author thanks Sue Robins for her inspiration and editorial guidance with this letter. 